\section{Correction to "Minimum Propagation Delays in VLSI"}

\section{C. Á. MEAD AND M. REM}

In the above paper, ${ }^{1}$ we wrote that because of

$$
N_{t}<N_{0} \beta^{-2 t} \text {, }
$$

Manuscript recelved August 29, 1983

The authors are with the Department of Computer Science, California Institute of

Technology, Pasadena, CA 91125
${ }^{1} \mathrm{C}$ A. Mead and M Rem, IEEE J. Sold-State Circults, vol SC-17, pp 773-775, Aug 1982 in which $N_{t}$ is the number of wires at level $i$, the number of wires must decrease exponentially with their length. As was pointed out by Eric Mjolsness, thes is an incorrect statement: the formula just states that the number of wires decreases exponentially with the level number. Since the total area is the same at each level and since the area of a wire increases as the square of its length, the number of wires must decrease as the inverse square of the wire length.

We apologize for any misconceptions our original statement may have caused. 